\title{
LE RESUME DOCUMENTAIRE: PROBLEMES ET SOLUTIONS*
}

\author{
LOUISE LARIVIERE
}

\begin{abstract}
This paper summarizes the different types of errors commonly made by students in the preparation of summaries and abstracts and proposes a methodology by which these errors can be eliminated in order to improve the quality of summaries and abstracts pertaining to documents. These errors are manifested not only in the process of abstraction itself, but also in the mechanics of structure, form and presentation. They constitute errors made at different stages of analysis and synthesis and are indicative of an absence or poor application of certain key activities or capacities.
\end{abstract}

\section{INTRODUCTION}

Beaucoup de professeures ${ }^{1}$ le disent: peu d'étudiantes réussissent à maîtriser la technique du résumé documentaire. ${ }^{2}$ La raison en est, bien souvent, que ces dernières ne savent pas quelle information pertinente extraire du document et où, à l'intérieur du texte, puiser cette information. Ce que ces mêmes professeures ne disent pas toujours, cependant, c'est qu'elles se contentent de fournir, bien souvent, une information succincte sur le médium et de présenter des consignes vagues et imprécises au moment de soumettre un travail sur le résumé. Certaines se limitent même à formuler le travail comme suit: "Vous me ferez, pour lundi prochain, un résumé de ce texte," en considérant comme acquis que les étudiantes savent

\footnotetext{
*Communication présentée lors du congrès annuel de l'Association canadienne des professeurs de rédaction technique et scientifique, dans le cadre des Sociétés savantes, Université de Windsor, Windsor (Ontario), 29-30 mai 1988.
} 
exactement ce qu'il faut faire. (Tout le monde sait ce que résumer veut dire, voyons!). Il ne faut donc pas s'étonner que, dans un tel cas, les résultats obtenus soient médiocres. Car il ne suffit pas de demander un résumé pour en recevoir un. Encore faut-il qu'on en ait, au préalable, engseigné les rudiments.

Au fait, qu'est-ce que résumer veut dire? Par nature, résumer c'est rendre l'essentiel du contenu d'un document ${ }^{3}$ sous forme d'un court texte limité en mots. L'action de résumer consistera done en une réduction et le résultat, en un document nouveau. Mais, d'abord, qu'entend-on par "l'essentiel" du contenu d'un document et comment le reconnaitre, cet essentiel? Que dire, ensuite, d'un "texte"; ce dernier ne constitue-t-il pas un tout autonome et cohérent qui répond a des lois strictes de composition?

À combien de "mots", finalement, doit-on se limiter et quel critère doit-on adopter pour définir un mot: critère graphique ou lexical? Ces trois questions trouvent leur réponse dans les deux fonctions qu'exerce le résumé et qui s'accomplissent a l'intérieur de leur démarche respective: l'analyse et la synthèse. La première fonction du résumé, c'est d'être une épreuve de compréhension ou de lecture qui s'effectue lors des deux étapes de l'analyse: le dépouillement du document et la sélection et la hiérarchisation des idées; la deuxième, une épreuve d'expression ou d'écriture qui s'effectue lors des trois étapes de la synthese: l'élaboration du plan, la rédaction proprement dite et la présentation.

Chacune de ces épreuves et des démarches qu'elles sous-tendent, suppose, par ailleurs, des capacités intellectuelles bien définies. Si l'une (ou plusieurs) d'entre elles fait défaut à une étape donnée d'exécution, diverses erreurs, affectant tantôt la nature et le fond, tantót la structure, la forme et la présentation du résumé, se produiront, les unes communes à tous les types de résumés (qu'ils soient de type sommaire, condensé ou synthèse ${ }^{4}$ ), les autres spécifiques aux résumés de type synthèse.

C'est donc un relevé de ces erreurs, au nombre de dix, que nous allons présenter et auxquelles nous tenterons d'apporter les correctifs nécessaires, dans le but d'améliorer la qualité des résumés documentaires.

\section{I - LES ERREURS D'ANALYSE}

Les erreurs de nature et de fond constituent essentiellement des manquements a la première démarche qu'est lanalyse, a l'étape du dépouillement ou a l'étape de la sélection et de la hiérarchisation, alors 
qu'une ou plusieurs activités ou qu'une ou plusieurs capacités ne se sont pas ou se sont mal exercées.

\section{A - LES ERREURS DE NATURE}

À l'étape du dépouillement ou analyse globale, deux erreurs de nature peuvent se commettre qui se caractérisent par l'excès, excès dans l'insuffisance: l'essoufflement ou omission; ou exces dans l'abondance: l'extrapolation ou addition, toutes deux attribuables a une méconnaissance de ce qu'est l'essence même du résumé.

\section{L'essoufflement ou omission}

L'essoufflement ou omission consiste à mettre un terme à la lecture du document original et, par voie de conséquence, a la rédaction du résumé une fois que la limite de mots, imposée dans les consignes, a été atteinte.

Une telle erreur est attribuable à deux choses: d'abord, au fait que l'on ne savait sans doute pas qu'il fallait lire et résumer le document en entier (Il y a de ces évidences dont il faut faire mention parfois!); puis, au fait que l'on a abordé la rédaction du résumé avant même d'avoir terminé la lecture du document. Il en résulte donc un résumé fragmentaire qui pèche par manque d'exhaustivité et qui "modifie les rapports entre les éléments" (Petroff, 1975: 55) puisque certains auront été omis.

Il serait assez facile de corriger cette erreur en donnant des consignes précises: $1^{0}$ ) lire, d'abord, le document en entier avant d'en entreprendre le résumé; $2^{\circ}$ ) résumer, ensuite, la totalité du document puisque c'est là une des exigences du médium de rendre compte de l'ensemble de son contenu.

\section{L'extrapolation ou addition}

Contrairement l'essoufflement, l'extrapolation ou addition se caractérise par la surabondance: ne pouvant se limiter, on déborde du contenu strict du document original pour faire part de des réflexions sur le sujet traité, ou pour s'étendre sur des considérations générales, ou encore pour faire des rapprochements avec des idées ou des faits connexes. Ce ne sont plus alors les idées de l'auteure qui sont exprimées mais des idées personnelles, totalement etrangeres au document. Il s'agit alors, dans ce cas, non plus d'un résumé de texte mais d'une explication de texte.

Cette trop grande liberté par rapport au document se manifeste bien souvent lorsque ce dernier traite d'un sujet controversé, d'actualité, ou de 
grandes questions qui touchent aux valeurs ou aux croyances profondes des individus. C'est aussi le fait, selon Logié et Mouchez (1970: 30), "d'étudiants dont les qualités d'expression et de culture sont remarquables." Une parfaite maftrise de la langue et une grande culture seraient-elles incompatibles avec le parfait maniement du résumé? Nous ne le croyons pas. L'extrapolation résulte plutôt d'un manque d'objectivité de fidélité ou de soumission au texte, et d'une trop grande "tentation" de faire valoir son point de vue, voire même, selon Petroff (1975: 55), "d'une tricherie fondamentale qui fait passer en fraude des informations attribuées au destinateur premier," i.e. l'auteure.

Pour pallier ces manques, il faudrait rappeler qu'il est dans la nature même du résumé de reproduire objectivement et fidèlement le contenu du document, non d'en faire une appréciation ni d'y apporter des jugements de valeur.

Consignes et rappels sur la nature même du résumé ne suffisent toutefois pas. D'une façon générale, il importe, pour contrer ces deux erreurs, de bien expliquer en quoi consiste cette première étape et d'y trouver des applications concrètes. Essentiellement, cette étape vise à faire saisir globalement le message du document. On y parvient en faisant examiner, dans un premier temps, la composition graphique du document: titre et intertitres, numérotation, nombre et disposition des paragraphes; en faisant relever, dans un deuxième temps, sa structure interne qui se révèle par les têtes de paragraphes, l'introduction et la conclusion, les charnières et les points de repere; en faisant dégager, dans un troisième temps, sa signification par un relevé des mots-clés, par la recherche du sens des mots incompris, par une simplification de la structure des phrases trop complexes. Une telle démarche réussira, ainsi, a freiner l'élan des étudiantes trop pressées qui résument prématurément ou trop exubérantes qui dissertent au lieu de résumer.

Bien sür, il y aurait lieu, par la suite, à la deuxième étape, d'insister sur la nécessité d'effectuer une analyse approfondie du document qui en fera ressortir les points essentiels, puis d'en dresser un plan précis et détaillé--et de s'y en tenir au moment de la rédaction du résumé.

\section{B - LES ERREURS DE FOND}

Les erreurs commises, a l'étape de la sélection, témoignent d'une incapacité de distinguer entre l'essentiel et l'accessoire, d'une part, et d'une incapacité d'effectuer, à partir d'un ensemble de données, les généralisations nécessaires, d'autre part, et d'atteindre, par le fait même, à un degré 
d'abstraction. Ces erreurs, très courantes, sont le découpage-montage, l'émiettement ou éparpillement, la paraphrase et l'effet coquetel.

\section{Le découpage-montage}

La première erreur de cette catégorie, le découpage-montage, consiste en la "technique", fort répandue, "des ciseaux et de la colle." Par ce procédé, on pige, ici et là, dans le document, des mots et des lambeaux de phrases (sans compter les phrases entières), puis on en effectue un habile montage en les mettant bout a bout.

Condenser ou juxtaposer, au petit bonheur, phrases et paragraphes sans en faire apparaître l'essentiel, en plus de témoigner d'une trop grande "servilité" par rapport au texte (Logié et Mouchez, 1970: 30), démontre clairement une inaptitude à l'analyse et à la généralisation, à moins que ce ne soit là le résultat d'une certaine paresse manifestée par des êtres qui se contentent de solutions de facilité. Une telle condensation mécanique et superficielle conduit, néanmoins, déformer l'image du document et à détruire la pensée originelle de l'auteure, puisque ce sont les phrases et les tournures qui sont contractées et non les idées (Moreau, 1984: 26). On risque, par ce rapiecçage de bribes, de ne pas rendre tout le contenu du document ou d'en éliminer les idées les plus riches, donc de passer à côté de l'essentiel et de nuire à la cohérence du document.

\section{L'émiettement ou éparpillement}

La deuxième erreur de cette catégorie, l'émiettement ou éparpillement, consiste, pour sa part, à s'attacher aux détails non significatifs du document plutớt que d'en dégager les idées dominantes. Encore ici, l'incapacité de distinguer entre l'essentiel et l'accessoire fait en sorte que le résumé produit manquera de généralité et de précision. De plus, étant donné que certains points majeurs n'auront pas été cernés ou auront été mis sur le même pied que les points accessoires, il manquera de substance et d'équilibre.

\section{La paraphrase}

Pour ce qui est de la paraphrase, troisieme erreur d'analyse, elle consiste en une reproduction servile du contenu du document, mais exprimée en des termes différents et de façon plus diffuse, bien souvent aussi linéaire.

Cette sorte de "verbiage" à propos du document, tout en étant un développement, ne va pas aussi loin que l'extrapolation dans l'explication et 
l'interprétation. D'une servilité aussi grande que le découpage-montage, bien que différente, il accuse, néanmoins, une incapacité d'extraire les idées du document, pour ensuite les généraliser et les agencer, a laquelle on supplée par du remplissage, caractéristique d'un manque de concision.

\section{L'effet coquetel}

La quatrieme erreur, contrairement aux trois premières, est spécifique aux résumés de type synthèse (soit les résumés portant sur plusieurs textes ou sur un dossier constitué de plusieurs pièces). L'effet coquetel, produit par le résumé, résulte du fait d'avoir bien mélangé, dans une même phrase ou dans un même paragraphe, diverses idées puisées, au hasard, dans l'un ou l'autre des documents à résumer. Tout comme pour le découpagemontage, c'est la cohérence de chacun des documents qui en sera atteinte de même que l'intégrité de chacun d'eux.

Toutes ces erreurs découlent finalement du fait que l'on cherche à résumer des mots et non des idées, alors qu'il est dans la nature du résumé de ne sauvegarder que l'idée du document et non son expression sous forme de "citations tronquees" (Baril et Guillet, 1981: 127). Pour éviter que de telles erreurs ne se produisent, il est nécessaire de bien faire saisir la nature et l'importance de cette deuxième étape de l'analyse qui se subdivise ellemême en deux sous-étapes: $1^{0}$ ) la sélection ou l'inventaire des idées (ou analyse détaillée); $2^{0}$ ) la hiérarchisation des idées.

C'est lors de cette étape qu'il convient, d'abord, de dégager P'essentiel des idées en décomposant le document "en ses éléments constitutifs" de manière a faire ressortir "les développements successifs de la pensée de l'auteur" (Thilliez, 1972: 13). Pour en arriver a "distinguer le contenu objectif de l'interprétation" (Goulet, 1987: 67), il est nécessaire de dépouiller le document de tout ce qu'on appelle les "variations du message" (Arambourou et al., 1974: 7) dans lesquelles réside le supplément d'informations: éléments purement descriptifs, illustratifs ou anecdotiques, pour ne retenir que l'information brute ou message premier ("degré zéro"), parfois exprimée clairement en début ou en fin de paragraphe, parfois a extraire d'une "masse" narrative.

De plus, il y aurait intérét à proposer des exercices précis qui visent a faire parvenir au degré d'abstraction et de généralisation souhaité. On pourrait, par exemple, a partir d'un long paragraphe, en faire relever les différentes catégories de pensée: points de vue, aspects, propriétés, modalités, qualités, actions, états, etc.; le faire résumer en une phrase dans des termes totalement étrangers au document; le faire hiérarchiser sous 
forme de plan détaillé comportant jusqu'à trois niveaux de subdivisions à l'intérieur de chacun desquels les idées seraient exprimées sous forme nominale.

Il convient, ensuite, d'établir une hiérarchie entre les idées qui n'ont pas toutes la même valeur afin de faire ressortir le cheminement suivi par l'auteure i.e. son plan. Pour ce faire, on retient le paragraphe comme point de départ de l'analyse et on en extrait les idées principales qui constituent les arguments du document et auxquelles se greffent les idées secondaires pour les expliciter. Une fois les idées principales sériées, on les compare en vue de regrouper en idées générales-i.e. en grandes phases de développement de la pensée--celles qui présentent une certaine unité de fond. On schématise, enfin, ces trois catégories d'idées sous forme de tableau synoptique divise en autant de colonnes afin de rendre compte du déroulement linéaire du document. (Thilliez, 1972: 15 et passim).

\section{II - LES ERREURS DE SYNTHESE}

Les erreurs de structure, de forme et de présentation constituent des manquements à la deuxième démarche qu'est la synthese, à l'une ou l'autre de ses étapes (élaboration du plan, rédaction proprement dite et présentation).

\section{C - LES ERREURS DE STRUCTURE}

À l'étape de l'élaboration du plan, nous relevons deux erreurs qui résultent essentiellement d'un manque de structuration: l'effet mosaique et la juxtaposition.

\section{L'effet mosaique}

L'effet mosaique se produit lorsque le résumé est constitué de phrases qui condensent, soit de façon linéaire ou discursive, soit de façon désordonnée, chaque paragraphe du document original sans que s'établissent des liens entre elles.

Cette incapacité d'enchainer les phrases nuit évidemment a la cohésion du texie qui n'apparaft pas alors comme un tout organique bien articulé mais comme une juxtaposition de pièces détachées. Stalloni (1983: 33) attribue ce manque d'unité et de liant à la suppression, au moment de l'analyse du document, des transitions, des illustrations et des effets de style qui lui donnaient son équilibre et son homogénéité. 
Le rappel de certaines règles élémentaires de composition s'impose donc pour atténuer ce défaut. D'abord, il est nécessaire d'insister sur le fait que le résumé est avant tout un texte rédigé qui ne laisse pas de place à un style télégraphique ou schématisé; ensuite, de revoir les procédés de liaison et de transition qui servent à nuancer les propos et a tisser une trame entre eux, en s'attachant particulièrement a ceux qui établissent des oppositions binaires entre les énoncés (ex.: d'une part et d'autre part); finalement, de revoir la formation des phrases complexes (relatives, complétives et conjonctives) qui, par la complémentarité et la dépendance qu'elles créent, resserrent l'expression et en assurent la cohésion.

\section{La juxtaposition}

La juxtaposition, qui affecte les résumés de type synthèse, s'apparente a l'effet mosaique. Ce ne sont plus, toutefois, des bouts de phrases qui sont accolés ou juxtaposés les uns aux autres sans liens mais des résumés entiers, distincts et indépendants, effectués a partir de chacun des documents originaux.

Ainsi, l'absence de rapprochements entre les idées des différents documents ne permet pas de les comparer. Il n'y a donc pas d'affrontement des idées mais simple amalgame, d'où, encore ici, un manque de cohésion et d'unité.

Comme l'effet mosaique et la juxtaposition résultent, tous deux, non pas d'une erreur de forme, comme on pourrait le croire, mais bien d'un manque évident de structuration, ce dernier pourra être compensé, dans le cas de l'effet mosaïque, par l'élaboration d'un plan de rédaction détaillé à partir de la hiérarchisation des idées effectué lors de la deuxième étape de l'analyse; et, dans le cas de la juxtaposition, par un inventaire des idées, distinct pour chacun des documents à résumer, suivi d'une hiérarchisation comparée des idées, pour en faire ressortir les ressemblances et les divergences, à partir de laquelle s'élaborera le plan de rédaction du résumé.

\section{D - LES ERREURS DE FORME}

Pour ce qui est des erreurs de forme, elles constituent aussi des manquements à la deuxième démarche qu'est la synthese, à l'étape, cette fois-ci, de la rédaction proprement dite du résumé.

Nous nous limiterons à exposer celles qui pèchent contre la clarté du résumé à cause d'un trop grand souci de concision, soit les erreurs de 
synthétisation. Certes, il est nécessaire d'être concis dans un résumé puisque le genre l'exige; mais il arrive qu'en voulant trop l'être, on nuise à son intelligibilite.

\section{La synthétisation}

La synthétisation se manifeste par l'utilisation de phrases passepartout qui pourraient, en fait, s'appliquer a tout document et non au contenu spécifique de l'un deux (ex.: "Les projections sont forcément boitteuses; les résultats, négatifs") et de trop de noms abstraits et de mots amalgames (ex.: "l'approche marketing", "la question santé"); ainsi que par le recours trop fréquent à certaines ponctuations: parenthèses, tirets, deuxpoints et par une surcharge de certains procédes syntariques: appositions, incises, emboitements.

Pour suppléer au style trop haché et au caractère trop hermétique du texte qui en découlent, il n'y aurait qu'à éviter les noms abstraits en les remplaçant par les verbes d'action correspondants et à utiliser les mots amalgames, les signes de ponctuation et les procédés syntaxiques mentionnés avec modération et équilibre. Quant aux phrases passe-partout, il s'agit de les remplacer par d'autres dont le contenu tient compte de la spécificité du document à résumer.

\section{E - LES ERREURS DE PRESENTATION}

Les erreurs de présentation résultent d'une non-observance des consignes, donc d'un manque de conformité, en l'absence desquelles tout le bláme repose, bien entendu, sur les professeures.

\section{La non-observance des limites imposées}

La grande erreur réside principalement dans la non-observance des limites imposees. Il importe donc d'indiquer clairement le nombre de mots (ex.: 150 ou 200 mots) ou le pourcentage de réduction exigé (ex.: de 10 à $20 \%$ du document original) de même que la marge de tolérance (ex.: 10\% en plus ou en moins) et les pénalisations pour les écarts.

Pour faciliter la comptabilité, on peut exiger que le nombre de mots soit indiqué la fin du résume. Encore faut-il que les étudiantes sachent comment compter les mots. Aux fins du résumé, on considere comme mot toute unité graphique séparée soit par un espace, soit par un signe orthographique (trait d'union et apostrophe). Ainsi "pomme de terre" et "chauve-souris", bien que constituant un seul mot lexical chacun, compteront 
pour trois mots et deux mots respectivement; "l'élève", évidemment, comptera pour deux mots.

Nous présentons, dans le tableau 1 qui suit, une typologie de toutes les erreurs relevées.

\section{CONCLUSION}

Ainsi, il ne suffit plus de dire aux étudiantes: "Faites-moi un résumé du texte suivant" pour obtenir un bon résumé. Désormais, il faudra leur soumettre un travail de résumé en ces termes: "Faites un résumé condensé du chapitre 1 du livre L'Article $23^{5}$ sous forme d'un texte rédigé de 200 mots en tenant compte des caractéristiques de contenu, de forme et de présentation propres au genre. Une marge de $10 \%$ sera observée au-delà de laquelle un point sur 20 sera enlevé pour toute tranche supplémentaire ou déficitaire de 10 mots. Pour les fautes d'orthographe et de grammaire, un demi-point sera enlevé jusqu'à concurrence de quatre points sur 20."

Bien entendu, il aura fallu, au préalable, bien expliquer en quoi consistent les "caractéristiques de contenu, de forme et de présentation propres au genre", de même qu'en quoi consiste un "mot" et avoir fourni une méthode qui respecte les démarches d'analyse et de synthèse, mise en pratique au moyen d'exercices appropriés.

$\mathrm{Si}$, après cela, les professeures disent encore que peu d'étudiantes réussissent à mấtriser la technique du résumé i.e. savoir se limiter avec précision et élégance, elles pourront toujours, dans une ultime mesure d'incitation, écrire en rouge sur les copies: "Qui ne sut se borner ne sut jamais écrire." (Boileau) 
Tableau 1: TYPOLOGIE DES ERREURS COURANTES

I - Erreurs d'analyse

A - Erreurs de nature

1. Essoufflement ou omission

manque d'exhaustivité

2. Extrapolation ou addition

manque d'objectivité manque de fidélité manque de soumission

B - Erreurs de fond
3. Découpage - montage
4. Émiettement ou éparpillement

manque de cohérence

manque de généralité manque de précision manque de substance manque d'équilibre

5. Paraphrase manque de concision

6. Effet coquetel (résumé synthèse) manque de cohérence II - Erreurs de synthèse

C - Erreurs de structure

\begin{tabular}{|l|l|l|}
\hline 7. Effet mosaique & $\begin{array}{l}\text { manque de cohésion } \\
\text { manque d'unité }\end{array}$ \\
8. Juxtaposition (résumé synthèse) & $\begin{array}{l}\text { manque de cohésion } \\
\text { manque d'unité }\end{array}$ \\
\hline D - Erreurs de forme & manque de clarté \\
\hline 9. Synthétisation & \\
\hline E - Erreurs de présentation & manque de conformité \\
\hline & 10. Non-observance des limites & \\
\hline
\end{tabular}


NOTES

1. Tous les noms d'agent ont été indiqués au féminin (sauf dans les citations) pour éviter la surcharge qu'entrâne l'utilisation des deux genres. Ces noms féminins désignent, cependant, aussi bien les hommes que les femmes.

2. Par résumé documentaire, nous entendons toute forme réduite d'un document écrit, tel qu'il est défini à la note 3, par opposition à toute autre forme qui condenserait un document non écrit (filmique, iconographique, cartographique, etc.)

3. Par document, nous entendons toute forme écrite: texte, dossier constitué de plusieurs pièces ou ouvrage entier.

4. Ces divers résumés s'inscrivent à l'intérieur d'une typologie que nous nous proposions, initialement, de présenter. Mais une telle entreprise aurait débordé largement le cadre de cette communication. Aussi, nous en ferons l'objet d'un exposé subséquent.

5. Jacqueline Blay. L'Article 23: les péripéries législatives et juridiques du fait français au Manitoba, 1870-1986. Saint-Boniface (Manitoba): Les Editions du Blé, 1987. 392 p.

\section{REFERENCES}

1. Arambourou, Ch. et al. (1972). Guide de la contraction de texte. Paris: Classiques Hachette. 185 p. (Faire le point).

2. Baril, Denis et Jean Guillet (1981). Techniques de l'expression orale et écrite, Tome I. 5e éd. Paris: Editions Sirey. 267 p. (Uni-Tech).

3. Goulet, Liliane (1987). "Le résumé de livre" in Cahier de méthodologie. $4^{e}$ éd. Montréal: Université du Québec a Montréal. p. 67-79.

4. Logié, Paul et Philippe Mouchez (1976). La technique du résumé. Paris: Cujas. 99 p.

5. Moreau, Jean A. (1984). La contraction et la synthese de textes. Paris: Nathan. 159 p. (Université, information, formation). 
6. Pétroff, André (1975). "Méthodologie de la contraction de texte". Langue francaise 26 (mai 1975): 41-55, "Techniques d'expression".

7. Stalloni, Yves (1983). Méthode de contraction et de synthèse de textes. $2^{\mathfrak{c}}$ éd. revue et corrigée. Paris: Edition Marketing. 191 p. (Ellipses).

8. Thilliez, Henri (1972). La rédaction d'affaires. Paris: Chotard et Associés éditeurs, $1972.218 \mathrm{p}$.

Louise Larivière (Desaulniers) est professeure agrégé à l'Ecole de traduction du Collège universitaire de Saint-Boniface (Université du Manitoba). 